\title{
Neuigkeiten zur Ablösung der geplanten Ablösung der In-Vitro-Diagnostika-Richtlinie durch die In-Vitro-Diagnostika-Verordnung (IVDR) 05/2022 und Plasmaversorgung in Deutschland/EU und Fortführung der konzertierten Aktion des BDDH mit den Selbsthilfegruppen
}

\author{
Christoph Sucker $^{1,2}$ Jürgen Koscielny ${ }^{3}$ \\ ${ }^{1}$ Medizinisches Versorgungszentrum (MVZ) COAGUMED \\ Gerinnungszentrum Berlin, Berlin \\ 2 Medizinische Hochschule Brandenburg Theodor Fontane, \\ Brandenburg an der Havel \\ ${ }^{3}$ Charité Universitätsmedizin, Berlin
}

Hamostaseologie 2022;42:81-83.

\section{Neuigkeiten zur Ablösung der geplanten Ablösung der In-Vitro-Diagnostika- Richtlinie durch die In-Vitro-Diagnostika- Verordnung (IVDR) 05/2022}

Planmäßig sollte am 26.05.2022 die bisher geltende In-VitroDiagnostika-Richtlinie durch die In-Vitro-Diagnostika-Verordnung (IVDR) ersetzt und an diesem Tag auch unmittelbar rechtlich wirksam werden. Wir hatten bereits auf die befürchteten erheblichen Auswirkungen auf die Labordiagnostik, auch im Bereich der Hämostaseologie, hingewiesen. Wie andere Berufsverbände hatte auch der Vorstand des BDDH eine Verschiebung der Novelle als erforderlich erachtet, um eine adäquate Labordiagnostik in Deutschland weiter gewährleisten zu können, da viele Aspekte der geplanten Novelle zu massiven Einschränkungen im Laborbereich geführt hätten - mit unabsehbaren schwerwiegenden Konsequenzen für die gesamte Diagnostik und zu befürchteten Auswirkungen auf die Patientenversorgung.

Diesbezüglich gibt es nun erfreuliche Nachrichten: Die europäische Kommission hat einen Vorschlag für ein Ergänzung (Amendment) zur IVDR verfasst und eine entsprechende Pressemitteilung herausgegeben. Demnach sollen die gemäß Novelle erforderlichen Änderungen für die Herstellung und Anwendung von in-vitro-Diagnostika nicht wie geplant ab dem 26.05.2022 in Kraft treten, vielmehr sollen
Address for correspondence PD Dr. med. Christoph Sucker, COAGUMED Gerinnungszentrum GmbH, Medizinisches Versorgungszentrum (MVZ), Tauentzienstraße 7b/c, 10789 Berlin, Germany (e-mail: cs@coagumed.de).

Übergangsfristen erheblich verlängert und das Inkrafttreten der verschiedenen Aspekte der Novelle um Jahre verschoben werden. Hiermit wird erfreulicherweise verhindert, dass es zu Lasten der Patienten im gesamten EU-Raum zu deutlichen Einschränkungen der Labordiagnostik kommt.

Als eine wesentliche Begründung der Verschiebung auch aufgeführt, dass es im Rahmen der weltweiten COVID19Pandemie zu einer starken Belastung der Gesundheitseinrichtungen gekommen ist, welche die Umsetzung der IVDR zum geplanten Zeitpunkt massiv erschwert hat.

Es bleibt zu hoffen, dass dies Signalwirkung auch für andere aktuell anstehende Änderungen im Gesundheitswesen hat, die zu einem extrem ungünstigen Zeitpunkt zu einer weiteren Belastung der Gesundheitseinrichtungen - Kliniken, Praxen und Labore - in Deutschland führen dürften: Die anstehenden weiteren Schritte der Digitalisierung im Gesundheitswesen führen zu einer massiven Mehrbelastung mit hohem Aufwand in den Einrichtungen, der zurzeit kaum zu bewältigen ist; hier bedarf es dringend einer Verschiebung, um eine adäquate Patientenversorgung auch unter den massiv erschwerten Pandemie-Bedingungen zu ermöglichen.

Des Weiteren problematisch ist für viele Einrichtungen die politisch verordnete Impfpflicht für alle Mitarbeiter in Gesundheitseinrichtungen, nach der jeder Mitarbeiter im Pflege- und Gesundheitswesen ab dem 15.03.2022 verpflichtend eine vollständige Corona-Impfung nachweisen muss.
(C) 2022. Thieme. All rights reserved. Georg Thieme Verlag KG,

Rüdigerstraße 14,

70469 Stuttgart, Germany
DOI https://doi.org/ 10.1055/a-1694-4640. ISSN 0720-9355. 
Kurzsichtiger Weise sind hier nicht nur die Mitarbeiter mit unmittelbarem Patientenkontakt, sondern auch Mitarbeiter im Laborbereich sowie Verwaltungsmitarbeiter betroffen. In manchen Einrichtungen der Patientenversorgung beträgt der Anteil ungeimpfter Mitarbeiter 20-30\%, entsprechend der aktuellen Impfquote der Bevölkerung. Demensprechend wurde diese Impfpflicht sehr kontrovers diskutiert. Klar ist, dass es durch die Impfpflicht zu einer weiteren Personalverknappung in Praxen, Kliniken und Laboratorien kommen kann, gerade dann, wenn bedingt durch die aktuelle weitere Welle der Corona-Pandemie mit einem erhöhten Patientenaufkommen in Kliniken und Praxen sowie mit einem erhöhten Probenaufkommen in Laboratorien zu rechnen ist. Hier besteht dringlicher Handlungsbedarf, um einem Kollaps wichtiger Strukturen der Krankenversorgung in Deutschland nach dem Stichtag zu verhindern.

\section{Plasmaversorgung in Deutschland/EU und Fortführung der konzertierten Aktion des BDDH mit den Selbsthilfegruppen}

Nach den aktuellen Zahlen der Charité ist in den vergangenen 10 Jahren der Verbrauch an Plasmapräparaten, insbesondere von Fibrinogen, PPSB, Humanalbumin, Fibrinkleber und der Immunglobuline, dramatisch angestiegen (Meldezahlen nach $\S 21$ Transfusionsgesetz (TFG) von 2010 bis 2020). Zudem hat in der letzten Dekade die Zahl von Blutspenden deutlich abgenommen, von 95 Spendern /1.000 Einwohner im Jahr 2011 auf 79 Spender/1.000 Einwohner im Jahr 2020. Das in Deutschland gewonnene Plasma wird aus Apherese und Vollblut gewonnen und vom Deutschen Roten Kreuz e.V. (35\%), von staatlich kommunalen Einrichtungen (6\%) und privaten Zentren (58\%) gesammelt. Fast 70\% des Plasmas für die in Deutschland verbrauchten Plasmaprodukte stammt aus den USA.

Die aktuellen Daten des PEI zeigen, dass im Jahr 2019 keine verringerte Spendenbereitschaft der Blut- und Plasmaspende in Deutschland vorliegt. Aktuelle Daten aus dem Jahr 2020 liegen nicht vor. Die Daten der Charité zeigen einen deutlich erhöhten Plasmaverbrauch zwischen 2010-2020, sodass selbst bei gleichbleibender Spendenbereitschaft die Plasmaversorgung zukünftig zumindest an der Charité eingeschränkt sein könnte. Da die Charité in dem Feld der Plasmaversorgung eines der größten Zentren in Deutschland ist, ist zu erwarten, dass auch in anderen Plasmazentren die Verbräuche und Spendenmengen sich ähnlich entwickeln. Die Plasmaversorgung ist in Deutschland noch weitestgehend gegeben, jedoch kommt es bereits bei Immunglobulinpräparaten zu Lieferengpässen deutscher Hersteller. Das plasmatische Gerinnungsfaktorenkonzentrat X war letztjährig monatelang nicht lieferbar.

Seit dem Jahr 2019 wird der Verbrauch aller Plasmaderivate in Krankenhäusern nach § 21 TFG nicht mehr erfasst, sodass vorlaufend nur noch Immunglobuline der jährlichen Erfassung unterliegen. Im Folgenden werden die Verbräuche von weiteren relevanten Plasmaderivaten, die zur Therapie nötig sind, nicht systematisch erhoben. In internen Erhebungen der Kliniken werden dennoch weiterhin die Daten der
Plasmaderivatverbräuche erhoben, um die Einkäufe für den eigenen Bedarf über die Krankenhausapotheke zu steuern. Da die Daten der Versorgung nicht systematisch erfasst werden, kann aus einem Engpass von Präparaten ein schwerwiegender Lieferengpass entstehen.

Zudem ist nach Angaben des PEI der Verbrauch von normalen Immunglobulinen von den Jahren 2011 auf 2016 von knapp $4.500 \mathrm{~kg}$ auf über $8.100 \mathrm{~kg}$, um beinahe $79 \%$, angestiegen. Angaben zu den Verbräuchen ab dem Jahr 2017 liegen derzeit nicht vor. Zudem ist ein deutlicher Anstieg des Importes von Immunglobulinen nach Deutschland zwischen 2011 und 2019 aus dem Europäischen Wirtschaftsraum um knapp 90\% sowie dem Nicht-Europäischen Wirtschaftsraum von über $170 \%$ vorzuweisen. Gleichzeitig ist ein sinkender Export der Immunglobuline aus Deutschland vom Jahr 2011 zum Jahr 2019 im europäischen Wirtschaftsraum um knapp 37\% festzustellen. Der Export im nicht-europäischen Wirtschaftsraum unterliegt im gleichen Zeitraum starken Schwankungen, sodass zuletzt knapp 20\% mehr Immunglobuline exportiert wurden. Das Deutsche Hämophilieregister (DHR) erfasst Informationen und Verbräuche von Patientinnen und Patienten mit angeborenen hämophilen Gerinnungsstörungen, während Verbräuche bei erworbenen Hämostasestörungen nicht erfasst werden; somit werden große Mengen des Verbrauches von Plasmaderivaten, z.B. Fibrinogen und Prothrombinkomplexpräparaten (PPSB) nicht ausreichend berücksichtigt.

Pro Jahr beläuft sich die Gewinnung von Plasma zur Fraktionierung auf rund 3 Millionen Liter in Deutschland. Die aktuellen Zahlen zur Blut- und Plasmaversorgung in Europa sind derzeit nur bis zum Jahr 2015 verfügbar, sodass hier keine klare Aussage zur aktuellen Versorgungslage getroffen werden kann. Deutschland deckt bisher 50\% der gesamten Plasmaproduktion der EU ab. Die Plasmaherstellung der restlichen EU-Länder liegt deutlich darunter, sodass die meisten EU-Länder stark vom Zukauf außereuropäischer Plasmaprodukte abhängig sind.

Eine schnelle Aktualisierung der Daten der letzten Jahre wird nötig sein, um konkrete Aussagen über den tatsächlichen Verbrauch von Plasmaprodukten machen zu können sowie eine bessere Koordination zu initiieren, um ein Defizit von Plasmapräparaten rechtzeitig entgegenzuwirken und schwerwiegende Versorgungsengpässe zu vermeiden

Die Selbsthilfegruppen sprechen sich für den Ausbau zur Akquirierung von Plasmaspender/innen aus. Ein Anknüpfungspunkt bietet die öffentliche Unterstützung durch Kampagnen für Plasmaspenden, um das Interesse in der Öffentlichkeit für einen angestiegenen Plasmaverbrauch zu sensibilisieren. Zudem sollen die Zentren zur Blut- und Plasmaspende ausgebaut und leichter zugänglich gemacht werden, um vermehrt Spenderinnen und Spender zu gewinnen.

Es sollte Transparenz über die Liefermengen nationaler und internationaler pharmazeutischer Unternehmen hergestellt werden, um deren zukünftige Pläne für die Plasmaherstellung in der Versorgung berücksichtigen zu können. Zudem müssen für eine gute Versorgung mit Plasmapräparaten zukünftig Lieferketten transparent aufgezeigt werden. 
Im Fokus stehen insbesondere die USA, die für den größten Anteil der Plasmagewinnung weltweit verantwortlich sind. Den aktuellen Daten der „Plasma Protein Therapeutics Association“ (PPTA) aus dem Jahr 2020 ist zu entnehmen, dass die Plasmasammlung der USA aufgrund der COVID-19-Pandemie im Jahr 2020 im Vergleich zum Jahr 2019 um 10-15\% zurückgegangen ist. Um einer Versorgungslücke von Plasmaprodukten in Deutschland vorzubeugen, sollten die aktuellen Spendenzahlen aus der USA und die Kosten von inn- und ausländischen Plasmaprodukten vorliegen. Diese Daten wären von den pharmazeutischen Unternehmen zu kommunizieren und einzuschätzen, sodass eine stärkere Überwachung generiert werden kann.

Es fallen derzeit vier Bereiche der Plasmaversorgung auf, die in der Zukunft eine stärkere Aufmerksamkeit benötigen, um Versorgungsengpässen entgegenzuwirken. Diese sind erneut an die über eine konzertierte Aktion des BDDH mit den Selbsthilfegruppen politisch zuständigen Stellen kommuniziert worden.
- Es werden aktuelle Daten für eine stärkere Transparenz bezüglich des Verbrauchs von Plasmapräparaten in Krankenhäusern benötigt, die durch die Institutionen BfArM und PEI eingeholt werden sollen.

- Die Spendenbereitschaft für Blut- und Plasmaspenden sollte in Deutschland stärker gefördert werden, um einen erhöhten Bedarf an Plasmaprodukten zu decken.

- Die Finanzierung und Verteilung von Plasmaprodukten muss transparenter werden, um die Entwicklungen antizipieren zu können.

- Die Produktionsplanung von Plasmapräparaten der pharmazeutischen Unternehmer muss transparent kommuniziert werden, um Versorgungseinbrüchen entgegenzuwirken.

Für den Vorstand der Deutschen Hämostaseologen Priv.-Doz. Dr. med. Jürgen Koscielny, Vorsitzender

Priv.-Doz. Dr. med. Christoph Sucker, Beisitzer des Vorstandes 